\title{
Flexible Silicon Photonic Transmitter with Segmented Modulator and 32 nm CMOS Driver IC
}

\author{
Tam N. Huynh, Nicolas Dupuis, Renato Rimolo-Donadio ${ }^{1}$, Jonathan E. Proesel, Doug M. Gill, \\ Christian W. Baks, Alexander V. Rylyakov ${ }^{2}$, Clint L. Schow ${ }^{3}$, William M. J. Green, Benjamin G. Lee \\ IBM T. J. Watson Research Center, Yorktown Heights, NY 10598 USA. \\ ${ }^{I}$ Now at Costa Rica Institute of Technology, Cartago 30101, Costa Rica. \\ ${ }^{2}$ Now at Coriant Advanced Technology Group, NY 10016, USA. \\ ${ }^{3}$ Now at University of California at Santa Barbara, CA 93106 USA. \\ tnhuynh@us.ibm.com
}

\begin{abstract}
We present a novel silicon photonic transmitter including 90nm CMOS segmented modulator co-packaged with low power $32 \mathrm{~nm}$ CMOS driver IC. Optical equalization is demonstrated for the first time with the multi-segment Mach-Zehnder modulator at $22 \mathrm{~Gb} / \mathrm{s}$.

OCIS codes: (130.4110) Modulator; (250.3140) Integrated optoelectronic circuits.
\end{abstract}

\section{Introduction}

Recently silicon photonics which leverages the widely deployed complementary metal-oxide semiconductor (CMOS) technology has been acquiring attention for optical communications in datacenters as well as access and metro networks. With the advantages of building on top of the long history of CMOS technology, silicon photonics would potentially provide a low cost, large scale integration, power efficient and small footprint solution [1,2] to cope with the exponential increase in data traffic. At the same time, there is a requirement to drive down power consumption of the electrical integrated circuit (IC) in the optical transceiver for higher power efficiency of the optical link. In this paper we present a novel transmitter design, which employs a silicon segmented Mach-Zehnder modulator (MZM) wire-bonded to a low power multi-channel 32nm CMOS driver IC. The segmented MZM structure allows equalization (EQ) to be performed in the optical domain, with demonstrated improvements in the BER performance of the optical link. Without the EQ, an error floor is observed at $22 \mathrm{~Gb} / \mathrm{s}$ while with the EQ error free $\left(\mathrm{BER}<10^{-12}\right)$ transmission has been achieved. The work here couples the advantages of segmented modulators - which reduce the device capacitance seen by each driver segment enabling extended modulation bandwidth - with the advantages of a novel EQ scheme that is demonstrated here. Whereas a segmented modulator driven by an equalized driver IC typically requires a driver with significantly more bandwidth than the modulating device, the scheme demonstrated here provides an equalized optical waveform that can overcome bandwidth limitations in the link, while employing drivers and devices with more evenly matched bandwidths.

\section{Driver IC and segmented modulator design}

The 6-channel driver IC was fabricated in IBM's 32nm SOI CMOS technology. Each channel has a similar architecture to the single-channel driver reported in [3], but modified for lower power and to achieve a better interface with the modulator. The input RF signal drives a first stage of cross-coupled CMOS inverters that amplify the signal to swing from Vss to VDD (1V). Subsequently, the level shifter provides two outputs swinging from Vss to $V_{D D}$ and from VDD to $V_{D D}(2 \mathrm{~V})$. These outputs drive a single stacked CMOS inverter chain. The 2-V $\mathrm{V}_{\mathrm{pp}}$ output differential signal of each channel will then drive each segment of the silicon modulator in a push-pull configuration. Each channel's core circuits occupy $20 \mu \mathrm{m} \times 100 \mu \mathrm{m}$. The segmented MZM was fabricated in IBM developed CMOS Integrated Nano Photonics technology [2]. The modulator has six isolated $200 \mu \mathrm{m}-$ long PN phase shifters in each arm and the measured capacitance of each segment including the pad was about $130 \mathrm{fF}$.

\section{Experiments and Results}

The experiment setup is illustrated in Fig. 1. The segmented MZM modulator and 6-channel driver IC were wirebond co-packaged on a custom printed circuit board (PCB). A pattern generator is employed to generate a PRBS-7 sequence. The signal is then split into six streams and amplified to provide sufficient driving signal to 6 channels of the driver IC. Electrical phase shifters are used to finely control the delay of the driving signals. The differential output of each channel of the IC drives each segment of the modulator in push-pull configuration. A distributed feedback (DFB) laser is used to provide input light at a wavelength of $1310 \mathrm{~nm}$. A polarization controller is placed before the modulator. The light is edge-coupled to the silicon modulator through single-mode tapered-lensed fibers positioned with 3-axis precision stages. DC biases are provided to the PCB through ribbon cables. $\mathrm{V}_{\mathrm{DD}}$ is biased at $1 \mathrm{~V}$ while $\mathrm{V}_{\mathrm{DD} 2}$ is biased at $2 \mathrm{~V}$. A source-meter is employed to bias the cathode of the modulator at $2.5 \mathrm{~V}$. Output 
light from the modulator is tapped $1 \%$ for power monitoring, while $99 \%$ passes through an optical amplifier (OA). The amplified signal then goes through a variable optical attenuator (VOA) and a 50/50 coupler before entering a reference receiver, which is a commercial $43 \mathrm{~Gb} / \mathrm{s}$ receiver with integrated trans-impedance amplifier (TIA). The differential electrical output signal from the receiver is then fed into the BER tester and the sampling scope. A signal generator is deployed to provide external clock for the pattern generator, the BER tester and scope trigger.

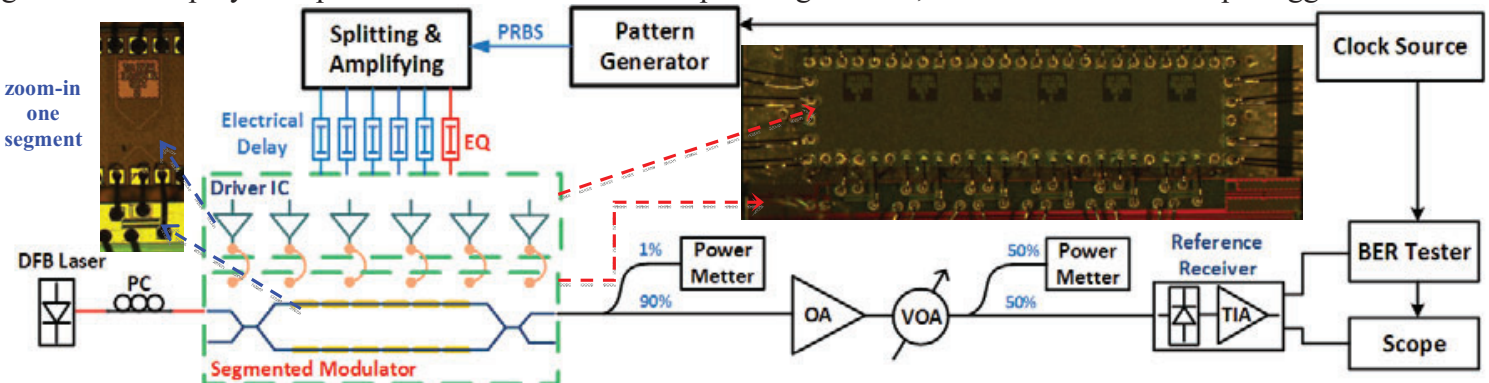

Fig. 1. Experiment setup with the inset micrographs of the silicon photonic transmitter including segmented modulator and CMOS driver IC.

To investigate the effects of optical EQ, five segments of the segmented modulator are driven by the same pattern with matched delay while the sixth segment is driven with reversed pattern and tuned delay to obtain feedforward EQ. By trading-off the optical extinction ratio of the transmitted signal, optical EQ would provide a more open eye that yields improvement in receiver sensitivity and jitter [4]. That is illustrated by the eye diagrams at $22 \mathrm{~Gb} / \mathrm{s}$ in Fig. 2(right) when the EQ is turned off and on. Measured extinction ratio (with $5 \mathrm{~Gb} / \mathrm{s}$ signal) is reduced from $2.2 \mathrm{~dB}$ to $1.8 \mathrm{~dB}$ when EQ is turned on. However, as shown in the jitter and BER measurement results in Fig. 2(left, center), the optical EQ significantly improved the jitter and receiver sensitivity of the link. At $22 \mathrm{~Gb} / \mathrm{s}$, without $\mathrm{EQ}$, the bathtub curve at $-3 \mathrm{dBm}$ received power in Fig. 2(left) is closed. When turning on the EQ, jitter measurement shows an open bathtub curve yielding estimated 0.21UI eye-opening at BER $=10^{-12}$ with $9.4 \mathrm{ps}$ deterministic jitter and 30 ps random jitter. The BER versus optical modulation amplitude (OMA) curves in Fig. 2(center) clearly confirm this improvement. Without EQ, an error floor appears at $10^{-10}$ while with $\mathrm{EQ}$, error free back-to-back transmission can be achieved with BER $=10^{-12}$ at $-7 \mathrm{dBm}$ OMA. The CMOS driver IC consumed low power at $105 \mathrm{~mW}$, with a corresponding power efficiency of $4.8 \mathrm{pJ} / \mathrm{bit}$.
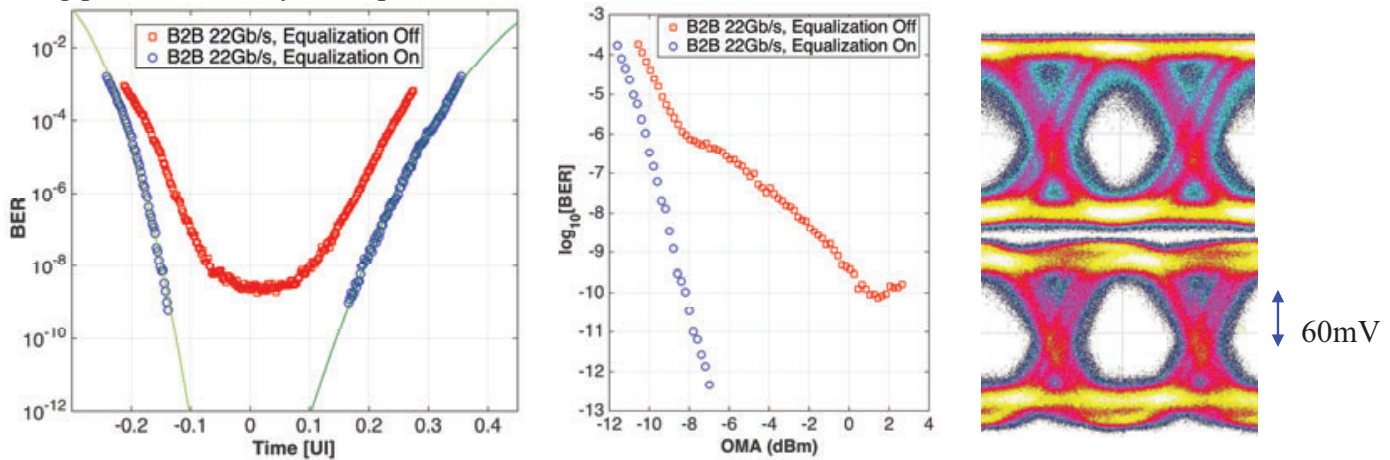

Fig. 2. Jitter measurement at $-3 \mathrm{dBm}$ received power (left) and receiver sensitivity (center) of segmented modulator at $22 \mathrm{~GB} / \mathrm{s}$ without/with optical EQ; Eye diagrams at 0dBm received power without (upper-right) and with (lower-right) optical EQ.

\section{Conclusion}

In this paper, we reported a silicon photonics segmented modulator fabricated with 90nm CMOS technology. The silicon modulator was driven by a 6-channel $32 \mathrm{~nm}$ CMOS driver IC wire-bonded in the same package. Error free transmission up to $22 \mathrm{~Gb} / \mathrm{s}$ was achieved with optical EQ. The transmitter further provides flexibility in varying the EQ's tap weight by altering the ratio of segments devoted to main and equalizing signals (5:1 here). Moreover the proposed transmitter configuration could be employed to generate signals with multilevel modulation format such as PAM-4, as has been demonstrated [5]. That shows the potential of a flexible silicon photonics transmitter for fast-reconfigurable agile optical networks.

[1] C.R. Doerr, "Silicon photonic integration in telecommunications," Front. Phys. 3:37, doi: 10.3389/fphy.2015.00037, 2015.

[2] N. Feilchenfeld et al., "An integrated silicon photonics technology for O-band datacom," IEDM 2015, paper 25.7.

[3] N. Dupuis et al., "30-Gb/s Optical Link Combining Heterogeneously Integrated ...," J. Lightw. Technol., 33, 657-662 (2015).

[4] A. V. Rylyakov et al., "Transmitter Predistortion for Simultaneous Improvements ...," J. Lightw. Technol., 30, 399-405 (2012)

[5] C. Xiong et al.,"A Monolithic 56 Gb/s CMOS Integrated Nanophotonic PAM-4 Transmitter," IEEE Optical Interconnects, paper MC3 (2015). 